Anaesthesist 2016 $65: 889-890$

DOI 10.1007/s00101-016-0243-7

Online publiziert:23. November 2016

๑) Springer Medizin Verlag Berlin 2016

CrossMark

\section{Gunther Pestel}

Klinik für Anästhesiologie, Universitätsmedizin der Johannes-Gutenberg Universität, Mainz, Deutschland

\title{
Höchstmaß an klinischer Kompetenz bei endovaskulären Eingriffen
}

Eine Investition in Wissen bringt immer noch die besten Zinsen.

(Benjamin Franklin)

Im Jahr 2014 verstarben in Deutschland 868.356 Personen. Wie in den vorigen Jahren war auch 2014 eine Erkrankung des Kreislaufsystems mit knapp $40 \%$ die häufigste Todesursache. Erkrankungen des Herzens und der Gefäße sind naturgemäß v. a. Erkrankungen bei älteren Menschen. Im Jahr 2014 waren $92 \%$ der an Erkrankungen des Kreislaufsystems Verstorbenen 65 Jahre und älter [10]. Die Anzahl der Menschen im Alter ab 65 Jahren wird in den nächsten Jahrzehnten absolut und relativ weitersteigen. Besonders stark wachsen wird diese Altersgruppe in den nächsten 20 Jahren durch den Eintritt der sog. Babyboomer ins Rentenalter. Während derzeit jede fünfte Person der Altersgruppe über 65 Jahren angehört, wird es 2060 jede dritte sein. Die Anzahl der über 80-Jährigen wird 2060 etwa doppelt so hoch sein wie heute: Vier von 10 Menschen im Alter ab 65 Jahren werden dann 80 Jahre und älter sein [11]. Die anästhesiologische Versorgung betagter Patientinnen und Patienten mit Herz- und/oder Gefäßerkrankungen wird unseren Berufsalltag zunehmend prägen.

Große periphere Gefäßeingriffe und Aortenchirurgie sind mit einem hohen kardialen Risiko behaftet [2]. Die v. a. bei offener Chirurgie auftretenden IschämieReperfusion-Phänomene können nicht nur zu unmittelbaren hämodynamischen Verwerfungen, sondern auch zu nachhaltigen Störungen durch Mediatorfreisetzung und Veränderung der Kapillarper- meabilität führen und erfordern daher die gesamte Aufmerksamkeit geschulter Anästhesisten.

\section{Rasante Entwicklung der Fallzahlen}

Auf der Suche nach Behandlungspfaden zur Reduktion des chirurgischen Traumas haben endovaskuläre Zugangswege zunehmend Bedeutung erlangt: Die perkutan endovaskuläre Versorgung bei peripherer arterieller Verschlusskrankheit (pAVK) hat sich in den vergangenen Jahren in Deutschland nahezu verdoppelt, die endovaskuläre Rekonstruktion bei abdominellen Aortenpathologien mehr als verdreifacht [14]. Die Vorteile eines endovaskulären Zugangs sind augenscheinlich:

- Die operative Exploration wird minimiert.

- Die hämodynamischen und metabolischen Auswirkungen einer chirurgischerseits durchgeführten Gefäßabklemmung werden (nahezu) ausgeschaltet.

- Gefäßanastomosen müssen nicht geknüpft werden.

An anästhesiologischen Verfahrensweisen stehen neben der Allgemeinanästhesie regionalanästhesiologische Ansätze sowie die Lokalanästhesie mit begleitender Sedierung zur Verfügung. Der Krankenhausaufenthalt wird deutlich verkürzt. Allerdings bedarf es großer Sorgfalt in der Patientenauswahl und einer akribischen perioperativen Planung, um Komplikationen zu vermeiden bzw. aufgetretene Komplikationen zu beherrschen [13]. Ernüchternderweise ist die perioperative Letalität beim endovaskulären Zugang zur Versorgung eines abdominellen Aortenaneurysmas zwar zunächst verkürzt [5], langfristig zeigt sich jedoch kein Unterschied im Vergleich zum offenen Eingriff $[1,12]$.

Somit besteht heute und in Zukunft für Anästhesisten die Notwendigkeit, in der Gefäßchirurgie sowohl bei offenen Operationen als auch bei elektiven endovaskulären Eingriffen ein Höchstmaß an klinischer Kompetenz abrufen zu müssen, um eine individuell patientenzentrierte perioperative Versorgung der ihnen anvertrauten Patientinnen und Patienten zu gewährleisten. Auch bei einer kontinuierlichen relativen Zunahme der endovaskulären Eingriffe im Vergleich zur offenen Operation wird sich diese Notwendigkeit nicht grundlegend ändern: Bei dringlichen Eingriffen und Notfalleingriffen (z. B. rupturiertes Aortenaneurysma) wird auch weiterhin die offene chirurgische Versorgung ihren Stellenwert behalten. Wird der offene Eingriff hingegen immer seltener, stellt sich für viele Krankenhäuser irgendwann die Frage nach der Aufrechterhaltung der Qualität in der klinischen Versorgung.

\section{Weitere Subspezialisierung in Anästhesie und Chirurgie nicht erstrebenswert}

Schlussendlich stellt sich nicht nur die Frage der Aufrechterhaltung der Expertise für die Erfahrenen in den Dienstgruppen von Chirurgie und Anästhesie, sondern auch die Frage der Weitergabe dieser Expertise an die nächste Generation [3]. Unangenehmerweise ist diese Problematik nicht nur auf das umschrie- 
bene Feld der Patientenversorgung in der Gefäßchirurgie beschränkt. Da eine ungebremste, immer weiter fortschreitende Subspezialisierung in Chirurgie und Anästhesie kein erstrebenswerter Endzustand sein kann, werden in der Fort- und Weiterbildung vielfältige Formen der klinischen Wissensvermittlung zwingend notwendig werden (Kooperation zwischen einzelnen Krankenhäusern, obligate Doppelbesetzung bei seltener werdenden komplexen Eingriffen im Sinne einer Mentor-Mentee-Ausbildung, Zuhilfenahme moderner Ausbildungstools wie „Full-scale“-Simulatoren usw.). Dass eine solche klinische Lehre nicht zum Nulltarif erhältlich sein wird, werden manche Interessenvertreter nicht hören wollen. Einleuchtend bleibt es trotzdem.

Anästhesiologischer Kompetenzgewinn und Kompetenzerhaltung sind ebenfalls zwingend für das zunehmend Bedeutung erlangende Themenfeld „anästhesiologisches Management bei endovaskulären Gefäßeingriffen“. Mit vorliegendem Leitthema stellen Rössel et al. eine ausführliche Betrachtung des $\mathrm{Ge}$ genstands für einen deutschsprachigen Leserkreis vor. Themenbezogen eingebettet in die Darstellung werden Betrachtungen zur allgemeinen Anästhesiologie (präoperative Vorbereitung, präoperative Diagnostik, Überwachung während der Eingriffe, Anästhesieverfahren). Detaillierte Beschreibungen der speziellen Anästhesiologie bei den mannigfaltigen endovaskulären Verfahren (neuroradiologische Interventionen, Eingriffe bei extrakraniellen Karotisstenosen, intrazerebrale Gefäßverschlüsse, Behandlung zerebraler Aneurysmata, endovaskuläre Interventionen der Aorta mit besonderer Berücksichtigung des Aortenaneurysmas, Anästhesie bei peripher-arteriellen Interventionen) bilden eine kundige Erörterung für interessierte Leser bzw. eine kompakte und übersichtliche Handreichung $\mathrm{zu}$ Beginn des Ausbildungsabschnittes. Die gewählten Abbildungen illustrieren sehr komplexe Sachverhalte anschaulich; in tabellarischen Darstellungen wird nötiges Faktenwissen präzise auf den Punkt gebracht.

\section{Mehr gute deutschsprachige Fachartikel!}

Nachdem sich Anfang des Jahrhunderts das Englische als „lingua franca" des Wissenschaftsbetriebes auch in Deutschland endgültig durchzusetzen schien [4], wurde unlängst die Bedeutung von Sprachdifferenzierung und -nuancierung sowohl für Deutsch [7] als auch für Englisch [8] herausgehoben. Diesseits internationaler Kongresse gibt es bei deutschsprachigen Chirurgen eine Präferenz für deutschsprachige Fachinformationen [9]. Es darf vermutet werden, dass sich diese Präferenz auch bei anderen Fachgruppen ähnlich findet. Gute deutschsprachige Fachpublikationen vertiefen also Wissen in der Breite, fundieren eine kritische Reflexion und erlauben eine verständige Umsetzung in der Praxis. Lehrende und Lernende schaffen gemeinsam die Lehre [6]. „Not fancy“ - aber beste akademische Tradition. Vielen Dank dafür nach Dresden.

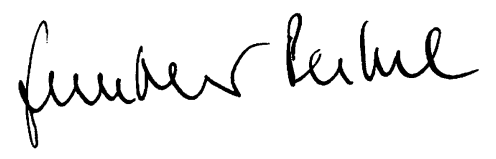

Gunther Pestel

\section{Korrespondenzadresse}

\section{PD Dr. G. Pestel}

Klinik für Anästhesiologie, Universitätsmedizin der Johannes-Gutenberg Universität Langenbeckstr. 1, 55131 Mainz, Deutschland pestel@uni-mainz.de

Interessenkonflikt. G. Pestel gibt an, dass kein Interessenkonflikt besteht.

\section{Literatur}

1. De Bruin JL, Baas AF, Buth J et al (2010) Longterm outcome of open or endovascular repair of abdominal aortic aneurysm. N Engl J Med 362:1881-1889

2. Deutsche Gesellschaft für Anästhesiologie und Intensivmedizin, Deutsche Gesellschaft für Innere Medizin, Deutsche Gesellschaft für Chirurgie (2010) Präoperative Evaluation erwachsener Patienten vor elektiven, nicht-kardiochirurgischen Eingriffen. Gemeinsame Empfehlung der Deutschen Gesellschaft für Anästhesiologie und Intensivmedizin, Deutschen Gesellschaft für Innere Medizin, Deutschen Gesellschaft für Chirurgie. Anaesthesist 59:1041-1050
3. Dyer JRWG, Jones P, Lim M (2008) Are training opportunities of abdominalaortic aneurysm repair being squandered? Anaesthesia 63:215

4. Falagas ME, Fabris E, Chelvatzoglou FC, Rellos $\mathrm{K}$ (2005) Penetration of the English language in science: the case of a German national interdisciplinary critical care conference. Crit Care 9:655-656

5. Greenhalgh RM, Brown LC, Kwong GP et al (2004) Comparison of endovascular aneurysm repair with open repair in patients with abdominal aortic aneurysm (EVAR trial 1), 30-day operative mortality results: randomised controlled trial. Lancet 364:843-848

6. Jaspers K (1946) Die Idee der Universität. Springer, Berlin

7. Lang GK (2010) Gibt es eine Zukunft für nicht-englischsprachige wissenschaftliche Zeitschriften? Klin Monbl Augenheilkd 227:601-602

8. Lyons G (2016) Language: another cause of publication bias. Eur J Anaesthesiol 33:620-621

9. Ronellenfitsch U, Klinger C, Buhr HJ, Post S (2015) Leseverhalten und Präferenzen hinsichtlich Abonnements wissenschaftlicher Fachzeitschriften. Chirurg 86:1051-1058

10. Statistisches Bundesamt (Hrsg) (2016) Gesundheit - Todesursachen in Deutschland 2014. Fachserie 12, Bd. 4. Statistisches Bundesamt, Wiesbaden, S Artikelnummer 2120400147004

11. Statistisches Bundesamt (Hrsg) (2015) Neue Bevölkerungsvorausberechnung für Deutschland bis 2060. Pressemitteilung 153/15. Statistisches Bundesamt, Wiesbaden

12. United Kingdom EVAR Trial Investigators, Greenhalgh RM, Brown LC, Powell JT et al (2010) Endovascular versus open repair of abdominal aortic aneurysm. N Engl J Med 362:1863-1871

13. Verhoeven ELG, Katsargyris A, Haulon S (2015) Caveat emptor: lessons learned from the endovascular treatment of complex aortic pathologies. Eur JVasc Endovasc Surg 49:363-365

14. Zimmermann A, Eckstein HH (2015) Der gefäßchirurgische Hybrid-Operationssaal - Standard in der invasiven Versorgung von Gefäßkrankheiten. ZGefässmed 12:16-21 